(C) 2018, The Authors. Published by FASS Inc. and Elsevier Inc. on behalf of the American Dairy Science Association ${ }^{\circledR}$.

This is an open access article under the CC BY-NC-ND license (http://creativecommons.org/licenses/by-nc-nd/4.0/).

\title{
Detection of microRNA in cattle serum and their potential use to diagnose severity of Johne's disease
}

\author{
Sandeep K. Gupta, ${ }^{, 1}$ Paul H. Maclean, $†$ Siva Ganesh, $†$ Dairu Shu, ${ }^{*}$ Bryce M. Buddle, ${ }^{*}$ D. Neil Wedlock, ${ }^{*}$ \\ and Axel Heiser* \\ *Animal Health, AgResearch, Hopkirk Research Institute, Grasslands Research Centre, Private Bag 11008, Palmerston North 4442, New Zealand \\ †Bioinformatics and Statistics, AgResearch, Lincoln Research Centre, Private Bag 4749, Lincoln 7608, New Zealand
}

\begin{abstract}
Mycobacterium avium subspecies paratuberculosis (MAP) causes Johne's disease in ruminants, which is characterized by chronic progressive granulomatous enteritis. The infection leads to wasting and weight loss in the animals and eventually death, causing considerable production losses to the agricultural industry worldwide. Currently available ELISA- and PCR-based diagnostic tests have limited sensitivity and specificity during early MAP infection in cattle, suggesting that there is an urgent demand for alternative diagnostic tests. Circulating microRNA (miRNA) have recently gained attention as potential biomarkers for several diseases in humans. However, knowledge and use of miRNA as biomarkers in diseases of ruminants, including Johne's disease, are very limited. Here we used NanoString nCounter technology (NanoString, Seattle, WA), a digital platform for amplification-free and hybridization-based quantitative measurement of miRNA in the sera of noninfected and naturally MAPinfected cattle with different severity of infection. Using probes developed against human miRNA, 26 miRNA were detected in cattle serum; 13 of these miRNA were previously uncharacterized for cattle. Canonical discrimination analysis using 20 miRNA grouped animals into 4 distinct clusters based on their disease status, suggesting that the levels of these miRNA can reflect disease severity. A model was developed using a combination of 4 miRNA (miR-1976, miR-873-3p, miR-520f$3 \mathrm{p}$, and miR-126-3p), which distinguished moderate and severely infected animals from noninfected animals. Our study demonstrated the ability of the NanoString nCounter technology to detect differential expression of circulating miRNA in cattle and contributes to widely growing evidence that miRNA can be used as biomarkers in infectious diseases in cattle.
\end{abstract}

Received March 20, 2018.

Accepted July 14, 2018.

${ }^{1}$ Corresponding author: sandeep.gupta@agresearch.co.nz
Key words: microRNA, Johne's disease, paratuberculosis, nanostring

\section{INTRODUCTION}

Johne's disease (JD) is caused by Mycobacterium avium subspecies paratuberculosis (MAP), which leads to diarrhea, wasting, and eventual death in ruminants. Johne's disease causes substantial losses to the global agricultural industry (Garcia and Shalloo, 2015). Based on MAP bacterial shedding in feces and other signs, JD is classified into 4 stages: silent, subclinical, clinical, and advanced (Whitlock and Buergelt, 1996; Clarke, 1997). Direct and indirect methods are used for diagnosis of MAP infection. Serology, fecal PCR, and IFN- $\gamma$ production are most commonly used for MAP detection and estimation of prevalence (Mortier et al., 2015; Smith et al., 2017). Performance of these diagnostic tests varies in early stages of MAP infection, when animals do not show any signs of the disease but shed MAP in feces. Alternative diagnostic tests to diagnose animals in early stages of the infection would be beneficial for improved herd management.

MicroRNA (miRNA) are short ( 22 nucleotides) noncoding RNA that regulate mRNA expression and protein translation in mammals (He and Hannon, 2004; Mehta and Baltimore, 2016). Body fluids, including plasma and serum, contain specific miRNA that are altered in various diseases (Wang et al., 2012). Because of their high stability in body fluids (Zhang et al., 2015), there is growing interest in using these circulating miRNA as biomarkers for progression of diseases in humans (Correia et al., 2017). MicroRNA have been proposed to play a crucial role in bovine immunity by regulating differentiation and function of immune cells and could be used as biomarkers in various diseases (Lawless et al., 2014; Dong et al., 2017). MicroRNA with differential expression have been reported in cattle in response to various pathogens, including bovine viral diarrhea virus, Mycoplasma bovis, and Staphylococcus aureus (Sun et al., 2015; Casas et al., 2016; Taxis et al., 
2017). Large repertoires of miRNA have been reported in the serum of cattle infected with MAP (Farrell et al., 2015; Liang et al., 2016; Malvisi et al., 2016). Together, these studies suggest that the abundance of circulating miRNA changes during infection and possibly correlates to severity of infection.

Currently, RNA sequencing, quantitative PCR, and microarray are used to detect and quantify miRNA. Sequencing of RNA is widely used to identify new miRNA (Pritchard et al., 2012; Tian et al., 2015). However, it is expensive and tedious and often does not allow accurate quantification of miRNA because of changes in transcript length and heterogeneous transcript distributions between the samples (Conesa et al., 2016). Another limiting factor for RNA sequencing is that the concentration of total RNA in body fluid is too low for most commercial library construction kits (Moldovan et al., 2014). Both RNA sequencing and quantitative PCR use enzymatic reactions (i.e., reverse transcription and polymerization), which contribute to variability. However, quantitative PCR is sensitive and cheap, can detect small changes, and can be used in high-throughput settings.

NanoString (Seattle, WA) technology is based on hybridization of sequence-specific probes to nucleic acids. It uses fluorescent molecular barcodes attached to the probes and single molecule imaging to count RNA copies (Geiss et al., 2008; Tsang et al., 2017). It has several advantages, such as amplification-free detection of miRNA and easy multiplexing of up to 829 human miRNA targets in 1 reaction, and it offers true population sampling by detecting direct miRNA molecules with $<0.5$-fold change sensitivity for target miRNA (Wang et al., 2016). Because a large number of mature mammalian miRNA share high sequence homology (Jin et al., 2009; Li et al., 2010), human miRNA probes are likely to detect miRNA of other mammalian species. Here we report the use of human miRNA probes developed by the Nanostring nCounter technology to measure abundance of miRNA in serum of cattle naturally infected with MAP. We also compared the expression levels of miRNA at different stages of JD and identified an miRNA profile that relates to disease severity.

\section{MATERIALS AND METHODS}

\section{Animals and Serum Samples}

All procedures involving the experimental use of animals were approved by the Grasslands Animal Ethics Committee, Palmerston North, Manawatu, New Zealand. A total of 24 Friesian, Jersey, or Friesian-Jersey cross cattle between 3 and 5 yr of age from various farms in the Manawatu region were used in the present study. The infected animals were culled based on poor body condition and milk production and were from herds with a history of JD on the farms, whereas the control animals were in average to good body condition and were culled based on declining milk production. At slaughter, multiple samples of the intestine, including the distal ileum, ileocecal valve, and mesenteric as well as ileocecal lymph nodes, were collected for histopathology and MAP culture. Infected or control animals were classified for severity of MAP infection on the basis of several pathophysiological conditions, including serology, histopathology score, and MAP bacterial culture as previously described (Shu et al., 2011). Briefly, histopathological changes from sections of the distal ileum and ileocecal valve were scored based on the condition of villi, cellular infiltrates in the lamina propria, the presence and severity of granulomatous lesions, and the acid-fast bacilli (AFB), whereas sections of the mesenteric and ileocecal lymph nodes were scored on the presence and severity of granulomatous lesions and the presence of AFB. Each parameter was scored separately from 0 to 3 . Histopathological scores of the 4 parameters (villi, cellular infiltration, granulomas, and AFB) for the distal ileum and ileocecal valve and 2 parameters (granulomas and AFB) for the mesenteric and ileocecal lymph nodes were added to obtain the combined score, which has been presented here. The tissue homogenate from the distal ileum, ileocecal valve, and mesenteric and ileocecal lymph nodes was cultured using Bactec 12B vials (Whittington et al., 1999; Nugent, 2011). The cultures registering a positive growth index $(>15)$ were also subjected to Ziehl-Neelsen staining to confirm the presence of mycobactin-dependent slow-growing AFB clumps as MAP. The animals were classified as MAP infected if MAP was cultured with presence of AFB clumps from 1 or more tissues and were graded as follows: $0=$ no growth or AFB in any of the tissues; $1=$ growth and AFB present only in 1 tissue; $2=$ growth and AFB present in 2 tissues; and $3=$ growth and AFB present in all of the tissues.

The serum samples used in the present study were from cattle at various stages of MAP infection from the previous study conducted in our group (Shu et al., 2011). We measured MAP-specific antibodies in the serum of these cattle by ELISA according to the manufacturer's instructions (Idexx Laboratories Inc., Westbrook, ME). Results for serology were expressed by an equation provided by the manufacturer:

$$
\begin{aligned}
& \text { sample/positive control } \%= \\
& 100 \times \frac{\text { sample OD } 450-\text { negative control OD } 450}{\text { positive control OD } 450-\text { negative control OD } 450} .
\end{aligned}
$$


where OD450 = optical density at $450 \mathrm{~nm}$.

Unknown samples were compared with those of positive and negative controls provided in the kit, and samples with sample-to-positive control percentage of $\geq 55 \%$ were considered positive as specified by the manufacturer. Based on pathophysiological conditions, including serology, histopathology score, and MAP bacterial culture, these animals were grouped into 4 disease groups - control (noninfected), mild, moderate, and severe - as shown in Table 1, which is based on the data from the previous paper (Shu et al., 2011).

\section{Comparison of miRNA Sequences}

The human miRNA v3 kit (NanoString, Seattle, WA) contains probes specific to 829 human miRNA along with probes against 5 nonmammalian miRNA. Sequences of these miRNA are given in Supplemental Table S1 (https://doi.org/10.3168/jds.2018-14785). Mature bovine miRNA sequences were retrieved from the miRBase (http://www.mirbase.org/; Griffiths-

Table 1. Grouping of Mycobacterium avium subspecies paratuberculosis (MAP)-infected animals based on their pathophysiology and MAP culture

\begin{tabular}{lcrcc}
\hline Group & $\begin{array}{c}\text { MAP-specific } \\
\text { IgG (OD) }\end{array}$ & S/P\% & $\begin{array}{c}\text { Granuloma } \\
\text { score }^{2}\end{array}$ & $\begin{array}{c}\text { MAP } \\
\text { culture }^{3}\end{array}$ \\
\hline Control & 0.09 & 0.38 & 4 & 0 \\
& 0.02 & 1.90 & 3 & 0 \\
Mild & 0.01 & 0.96 & 7 & 0 \\
& 0.01 & 0.73 & 3 & 0 \\
& 0.08 & -0.83 & 4 & 1 \\
Moderate & 0.01 & 0.17 & 5 & 1 \\
& 0.09 & 0.85 & 2 & 1 \\
& 0.08 & 0.14 & 3 & 1 \\
& 2.19 & 231.09 & 20 & 2 \\
& 1.36 & 139.83 & 18 & 2 \\
Severe & 2.00 & 210.34 & 13 & 2 \\
& 2.49 & 264.46 & 23 & 2 \\
& 2.39 & 252.79 & 18 & 2 \\
& 2.30 & 243.62 & 20 & 2 \\
& 2.73 & 290.56 & 23 & 2 \\
& 2.92 & 272.75 & 27 & 2 \\
& 2.45 & 259.54 & 31 & 3 \\
& 2.50 & 264.77 & 33 & 3 \\
& 2.82 & 262.95 & 35 & 3 \\
& 1.96 & 180.41 & 34 & 3 \\
& 2.37 & 219.41 & 34 & 3 \\
& 2.22 & 234.25 & 30 & 3 \\
& 2.05 & 215.77 & 29 & 3 \\
& 3.16 & 338.03 & 36 & 3 \\
\hline
\end{tabular}

${ }^{1} \mathrm{~S} / \mathrm{P} \%=100 \times($ sample OD450 - negative control OD450)/(positive control OD450 - negative control OD450).

${ }^{2}$ Combined score of the 4 parameters (villi, cellular infiltration, granulomas, and acid-fast bacilli) for the distal ileum and ileocecal valve and 2 parameters (granulomas and acid-fast bacilli) for the mesenteric and ileocecal lymph nodes.

${ }^{3}$ Based on MAP bacterial growth in 1 or more tissues using Bactec $12 \mathrm{~B}$ vials (Whittington et al., 1999; Nugent, 2011) and number of acid-fast bacilli clumps present in Bactec 12B cultures.
Jones, 2004, 2008; Kozomara and Griffiths-Jones, 2014), a biological database for miRNA sequences and annotations. To evaluate whether human miRNA probes could be used to detect cattle miRNA, sequences of the miRNA probes and bovine miRNA were compared using the BLASTN (Camacho et al., 2009) feature of Geneious 10 software (Biomatters Ltd., Auckland, New Zealand) with a word size of 7 and no complexity filtering and other settings set to default.

\section{RNA Isolation}

Total RNA was isolated from cattle serum using the Qiagen (Hilden, Germany) miRNeasy kit following the manufacturer's instructions. Briefly, $250 \mu \mathrm{L}$ of serum was mixed with 5 volumes of QIAzol lysis reagent. Three synthetic oligonucleotides (ath-miR159a, celmiR-248, and osa-miR414; Integrated DNA Technologies, Coralville, IA) were added at $400 \mathrm{fM}$ before RNA extraction as spike-in controls. The RNA was eluted in $200 \mu \mathrm{L}$ of RNase- and DNase-free water. The RNA was then concentrated to a final volume of $15 \mu \mathrm{L}$ using the salt and RNase-free glycogen (Sigma, St. Louis, MO) precipitation method as previously described (Gupta et al., 2016). The quality of the isolated miRNA was analyzed by running total RNA on a small RNA chip using a microfluidics-based system, the 2100 Bioanalyzer (Agilent Technologies, Waldbronn, Germany).

\section{miRNA Detection Using nCounter}

Detection of miRNA was performed using the nCounter (NanoString). We analyzed RNA samples for miRNA using a human miRNA kit v3 consisting of probes specific for 829 miRNA according to the manufacturer's instructions (NanoString). The reaction was carried out with $3 \mu \mathrm{L}$ of the total RNA isolated from cattle serum. A specific miRTag (NanoString) was ligated onto $3^{\prime}$ end of each mature miRNA in the sample according to the manufacturer's instructions. The reaction was incubated with ligation cleanup enzyme for 1 $\mathrm{h}$ at $37^{\circ} \mathrm{C}$ to remove unligated miRTags by enzymatic degradation. Five microliters of each miRNA-miRTag sample was hybridized to reporter code sets (in $20 \mu \mathrm{L}$ of hybridization buffer) and $5 \mu \mathrm{L}$ of capture code sets at $65^{\circ} \mathrm{C}$ for $18 \mathrm{~h}$. Once the hybridization was completed, the robotic nCounter Prep Station (NanoString) was used to remove the excess probes using 2-step magnetic bead-based purification, and samples were transferred to a cartridge. Color-coded miRNA-probe hybrids attached on the surface of the cartridge were counted on an nCounter digital analyzer (NanoString) and tabulated for each target molecule. 


\section{Data Analysis for miRNA Expression}

Tabulated data were retrieved from the analyzer as reporter code count files. The raw reporter code count files were imported into nSolver Analysis Software version 2.5 (http://www.nanostring.com/products/ nSolver) for analysis. Using the reporter library file (NS_H_miR_v3a) that contained the corresponding CodeSet information, nSolver's quality control routine was performed to flag the samples for exclusion according to the following parameters: fields of view registration $<75 \%$; binding density outside the 0.05 to 2.25 range; positive control linearity: positive control $\mathrm{R}^{2}$ value $<0.95$; and positive control limit of detection: $0.5 \mathrm{f} M$ positive control $\leq 2 \mathrm{SD}$ above the mean of the negative controls. With these settings, no sample was flagged and all samples passed the quality control.

To minimize the background from the data, the number of counts from the highest negative control +2 $\mathrm{SD}$ was subtracted from all the miRNA counts. After subtraction, the counts for individual miRNA were normalized against geometric mean counts of the 3 spike-in oligonucleotides. The miRNA that on average were expressed below the threshold of 15 copies did not show differences between groups and were not included in further analysis.

\section{Statistical Analysis}

Statistical analysis was performed using ANOVA on the number of normalized miRNA counts to identify the difference between groups of samples using Minitab Statistical Software version 17 (Minitab Inc., State College, PA). $P$-values $<0.05$ were considered statistically significant (with smaller values providing stronger evidence for significance), and $P$-values $\geq 0.05$ and $<0.10$ were considered a trend. To examine whether the 4 different disease groups could be separated based on overall miRNA expression, a canonical discrimination analysis (CDA) was performed on the miRNA in serum samples using the candisc package version 0.8 (Friendly and Fox, 2010) in $\mathrm{R}$ version 3.4.2 (https://www.R -project.org/). Canonical discrimination analysis works on a similar function to principal component analysis (PCA), where both analyses create variables that are linear combinations of the original variables. However, unlike PCA, CDA generates these linear combinations with respect to the groupings of individuals as opposed to the maximization of variation in dimensions. The resulting combinations of variables, called canonical discriminant functions (CDF), maximally separate the groups of individuals while keeping the variation within groups as small as possible. The coefficients of the CDF are listed in Supplemental Table S2 (https: //doi.org/10.3168/jds.2018-14785), with each CDF being represented as a column (i.e., CDF1 $=-10.01$ $\times$ hsa_miR_1976 + -5.07 hsa_miR_1246 + . . . + $20.56 \times$ hsa_miR_1290). The canonical discriminant scores were extracted from the output of the candisc function from the candisc package and examined using scatterplots to determine how well the treatments were separated.

Canonical correlation analysis (CCA) was conducted using the mixOmics package (Rohart et al., 2017) in R to examine the multivariate associations between 2 sets of variables, pathophysiological conditions (serology, histopathology score, and MAP bacterial culture) of the animals and the expression levels of 20 miRNA with most variance in the expression. Canonical correlation analysis seeks linear functions of one set of variables that are highly correlated with linear functions of the second set of variables. These correlations are known as canonical correlations. The canonical correlation scores for each animal were extracted from the output of the regularized canonical correlation function in the mixOmics package and explored via scatterplots.

\section{Development of Diagnostic Models}

Two models were developed using 4 miRNA (miR1976, miR-873-3p, miR-520f-3p, and miR-126-3p) with the significant maximum differential expression (Table 2 ). The first model was generated based on the difference between the expression of 2 miRNA with increased levels and 2 miRNA with decreased levels in the serum according to the formula (miR-1976 + miR-873-3p) (miR-520f-3p + miR-126-3p). The second model was generated based on the ratio of the expression of 4 miRNA according to the formula (miR-1976 + miR873-3p)/(miR-520f-3p + miR-126-3p). The ability of the combinations of miRNA to distinguish any of the status of disease severity was tested for significance using ANOVA and percentage separation statistics from CDA summaries according to the first model. The second model was tested using only ANOVA to significantly separate the animals based on their disease status and not CDA because CDA does not allow comparison of values presented as in ratios.

\section{RESULTS}

\section{MiRNA Sequence Comparison and Quality Control}

A comparison of known mature bovine miRNA sequences from the miRBase with sequences of the 829 human miRNA probes revealed an exact match for 180 bovine miRNA with the human miRNA probes, 
Table 2. Fold change in expression levels of microRNA in sera ${ }^{1}$

\begin{tabular}{|c|c|c|c|}
\hline \multirow[b]{2}{*}{ MicroRNA } & \multicolumn{3}{|c|}{ Fold change over control } \\
\hline & Mild & Moderate & Severe \\
\hline miR-1976 & 1.04 & 1.03 & $1.31^{* *}$ \\
\hline miR-1246 & 1.54 & 1.45 & 3.61 \\
\hline $\operatorname{miR}-4516$ & 1.68 & 0.44 & 3.08 \\
\hline miR-520f-3p & 0.96 & $0.54^{* * *}$ & $0.68^{*}$ \\
\hline miR-1268a & 0.95 & $0.07^{*}$ & 0.92 \\
\hline miR-612 & 0.75 & 0.77 & 0.67 \\
\hline miR-451a & 1.54 & 0.59 & 1.08 \\
\hline miR-1224 & 0.36 & 0.13 & 0.36 \\
\hline miR-126-3p & 0.72 & $0.35^{*}$ & 0.49 \\
\hline miR-192-5p & 0.81 & 1.00 & 0.97 \\
\hline miR-873-3p & 1.32 & 1.10 & $1.78^{*}$ \\
\hline miR-223-3p & 1.43 & 1.23 & 3.61 \\
\hline miR-6721-5p & 0.94 & 1.02 & 1.64 \\
\hline miR-363-3p & 1.09 & 1.20 & 1.69 \\
\hline $\operatorname{miR}-4532$ & 1.85 & 1.79 & 5.51 \\
\hline miR-598-3p & 1.04 & 1.05 & 1.39 \\
\hline miR-142-3p & 0.84 & 0.64 & 1.99 \\
\hline miR-4454 & 1.09 & 0.95 & 1.67 \\
\hline let-7b-5p & 1.00 & 0.90 & 1.43 \\
\hline miR-107 & 0.96 & 1.34 & 1.80 \\
\hline miR-1299 & 0.71 & 0.93 & 0.80 \\
\hline miR-642a-3p & 0.90 & 0.55 & 1.43 \\
\hline miR-188-5p & 0.94 & 0.67 & 1.07 \\
\hline miR-1915-3p & 1.18 & 0.31 & 1.82 \\
\hline miR-23a-3p & 0.83 & 0.50 & 1.03 \\
\hline miR-1290 & 0.95 & 0.67 & 3.61 \\
\hline
\end{tabular}

${ }^{1}$ Fold changes were calculated between samples in each of the disease groups and the control group.

${ }^{*} P<0.05,{ }^{* *} P<0.01,{ }^{* * *} P<0.001$ calculated using ANOVA.

indicating the suitability of the kit for the detection of miRNA in cattle.

The quality and integrity of small RNA in the total RNA isolated from serum were evaluated using an Agilent small RNA chip (Agilent Technologies). This chip also provides relative abundance of small RNA to the total RNA for each sample. The results showed an average abundance of $82 \%$ of small RNA in the total RNA isolated from serum (Supplemental Figure S1; https:// doi.org/10.3168/jds.2018-14785).

To detect small changes in individual miRNA, synthetic oligonucleotides were added for volume- and quantity-based normalization. Therefore, miRNA from 3 nonmammalian species (synthetic oligonucleotides corresponding to ath-miR159a, cel-miR-248, and osamiR414) were added as spike-in controls during RNA isolation. In addition to 829 human-specific miRNA probes, the human miRNA kit v3 also contains probes for these oligonucleotides.

\section{Detection of miRNA in Serum of Cattle Infected with MAP}

Using the human miRNA kit v3 developed by the NanoString nCounter technology, 26 miRNA were de- tected in cattle serum (Table 2). Five of these miRNA were identical in humans and cattle (Table 3). A further 8 had sequences homologous to bovine miRNA with a 1- to 2-nt mismatch (Table 3). Interestingly, the other 13 detected miRNA have not yet been described in cattle (Table 4). These results show that probes specific to human miRNA can be used to detect miRNA in cattle.

\section{Differential Expression of miRNA in Serum of Cattle Infected with MAP}

Several miRNA were found to be differently expressed among the 4 disease stage groups (Figure 1). Although the expression of some of the miRNA was increased with severity of the disease, some showed decreased expression with the disease progression. Across all the samples, maximum number of counts was obtained for miRNA-1976 and miRNA-1246 (Figure 1).The expression of miRNA-1976 was increased by 1.3 -fold $(P<$ 0.01 ) in serum of the severely infected animals compared with the controls. The levels of miRNA-873-3p were also increased by 1.7 -fold $(P=0.04)$ in serum of the severely infected animals compared with the control animals. A trend was observed in the expression of miRNA-1246, with a 3.6-fold increase in the severely infected cattle compared with the control animals $(P$ $=0.09)$. In addition, miRNA-233-3p showed a trend of increased expression $(P=0.10)$ in the severely infected animals compared with the control animals.

The expression of miRNA-520f-3p was decreased in moderately and severely infected serum by 0.5 -fold $(P<$ $0.001)$ and 0.7 -fold $(P=0.03)$, respectively, compared with the control animals. The expression of miRNA126-3p and miRNA-1268a was found to be decreased by 0.35 -fold $(P=0.04)$ and 0.07 -fold $(P=0.03)$, respectively, but only in moderately infected animals compared with the control group. A trend of decreased expression $(P=0.09)$ was also observed for miRNA$126-3 p$ in the severely infected animals compared with the control animals.

\section{Relationship Between miRNA Expression, Disease Severity, and Pathophysiological Condition}

To evaluate whether MAP infection in cattle had any combined effect on changes observed in the levels of miRNA, 20 of the miRNA with the most variance in differential expression were used to perform a CDA. The plot of corresponding canonical discriminant scores for the first $2 \mathrm{CDF}$ showed that all 4 groups clustered separately (Figure 2). Severe and control groups were clearly separated along the first CDF dimension, and both were separated from mild and moderate groups. 
Table 3. Human and bovine microRNA with matched sequence

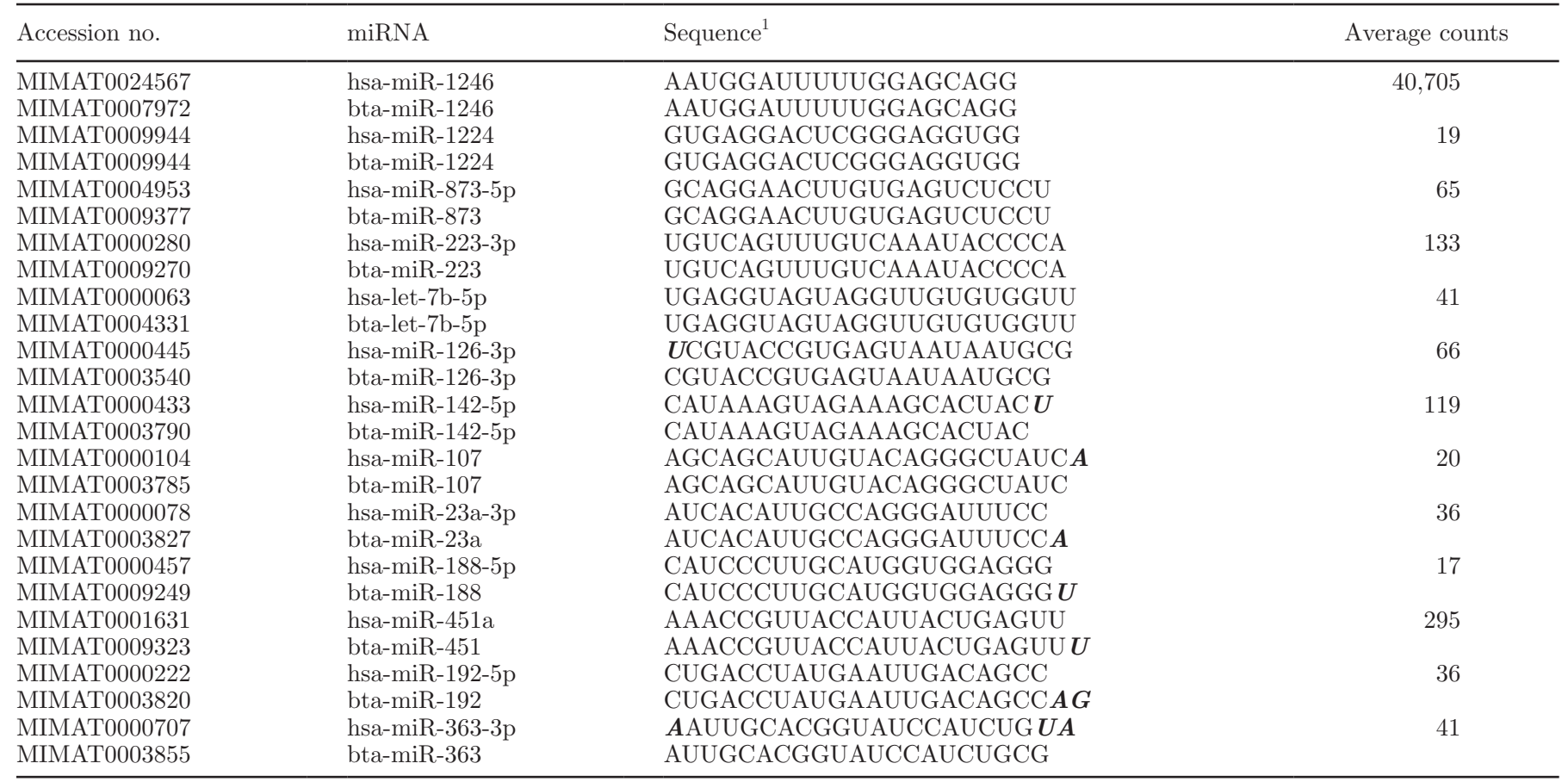

${ }^{1}$ Mismatched nucleotides are presented in bold and italics.

This separation accounted for $95 \%$ of the differences among the groups. The mild group was separated from the other groups along the second CDF (Figure 2).

The CCA between the expression of 20 miRNA and the pathophysiological conditions (serology and histopathology score) and MAP culture showed that miRNA-1976, miRNA-107, and miR-873-3p positively correlated with histopathology score (Figure 3). On the other hand, miRNA-520f-3p, miRNA-1224, and miRNA-126-3p negatively correlated with histopathology score (Figure 3). A similar correlation was also observed with serology and expression of these miRNA.

\section{Diagnostic Model Based on miRNA Expression}

Two models were generated and assessed to examine the potential of 4 miRNA (miR-1976, miR-873-3p, miR-520f-3p, and miR-126-3p) as biomarkers and to predict disease status of animals based on their expression levels in the sera. The first model allowed clear separation of severely infected animals from the control animals $(P=0.01)$, although no difference was observed between the mild and the control animals (Figure 4A). Furthermore, CDA on the combination of these $4 \mathrm{miR}$ NA separated moderate and severely infected animals

Table 4. Newly detected microRNA in cattle serum

\begin{tabular}{lllr}
\hline Accession no. & miRNA & Sequence & Average counts \\
\hline MIMAT0009451 & hsa-miR-1976 & CCUCCUGCCCUCCUUGCUGU & 10,830 \\
MIMAT0019053 & hsa-miR-4516 & GGGAGAAGGGUCGGGGC & 471 \\
MIMAT0002830 & hsa-miR-520f-3p & AAGUGCUUCCUUUUAGAGGGU & 205 \\
MIMAT0018976 & hsa-miR-4454 & GGAUCCGAGUCACGGCACCA & 110 \\
MIMAT0003280 & hsa-miR-612 & GCUGGGCAGGGCUUCUGAGCUCCUU & 61 \\
MIMAT0025852 & hsa-miR-6721-5p & UGGGCAGGGGCUUAUUGUAGGAG & 54 \\
MIMAT0019071 & hsa-miR-4532 & CCCCGGGGAGCCGGCG & 49 \\
MIMAT0003266 & hsa-miR-598-3p & UACGUCAUCGUUGUCAUCGUCA & 39 \\
MIMAT0005922 & hsa-miR-1268a & CGGGCGUGGUGGUGGGGG & 30 \\
MIMAT0005880 & hsa-miR-1290 & UGGAUUUUUGGAUCAGGGA & 24 \\
MIMAT0020924 & hsa-miR-642a-3p & AGACACAUUUGGAGAGGGACC & 20 \\
MIMAT0005887 & hsa-miR-1299 & UUCUGGAAUUCUGUGUGAGGGA & 16 \\
MIMAT0007892 & hsa-miR-1915-3p & CCCCAGGGCGACGCGGCGGG & \\
\hline
\end{tabular}



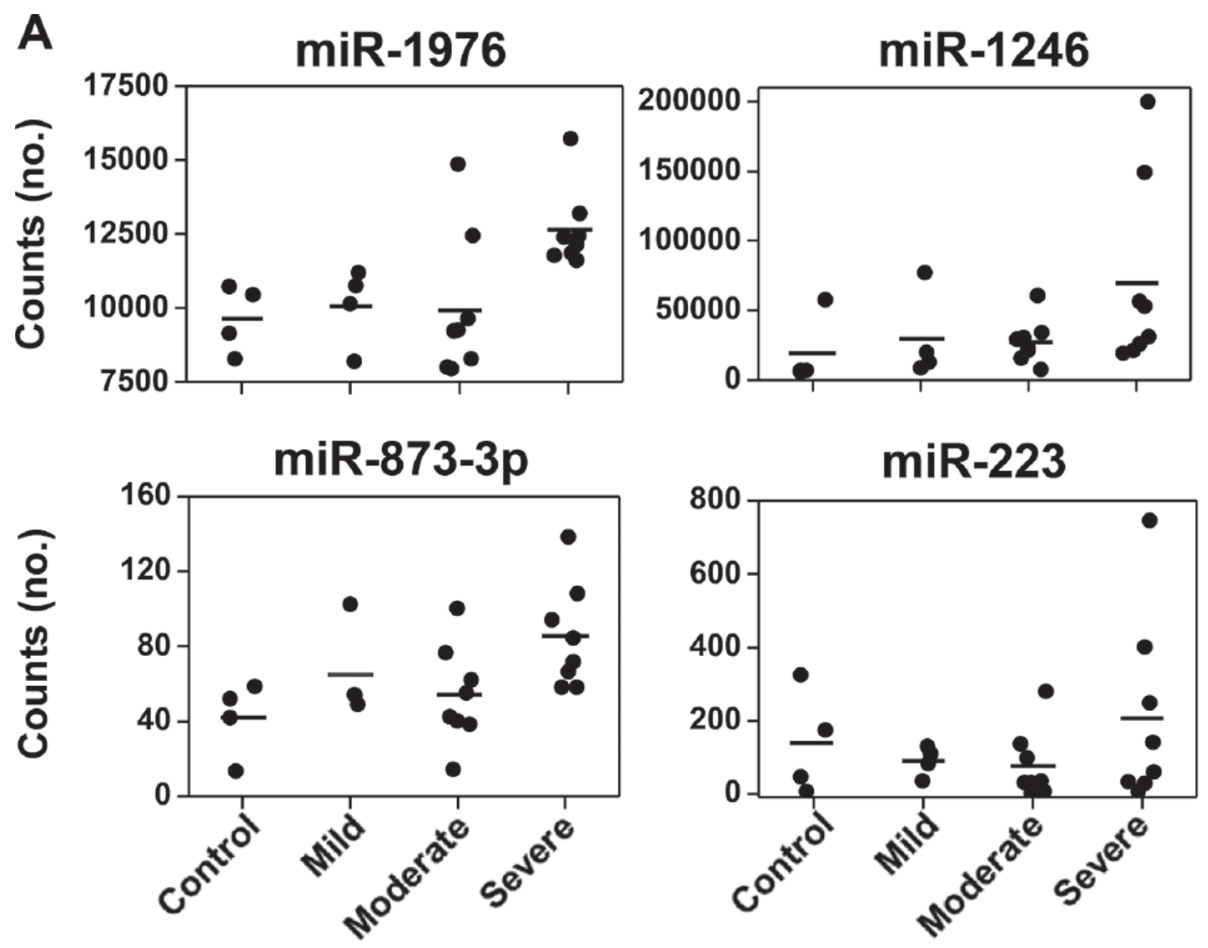

Upregulated miRNA in cattle
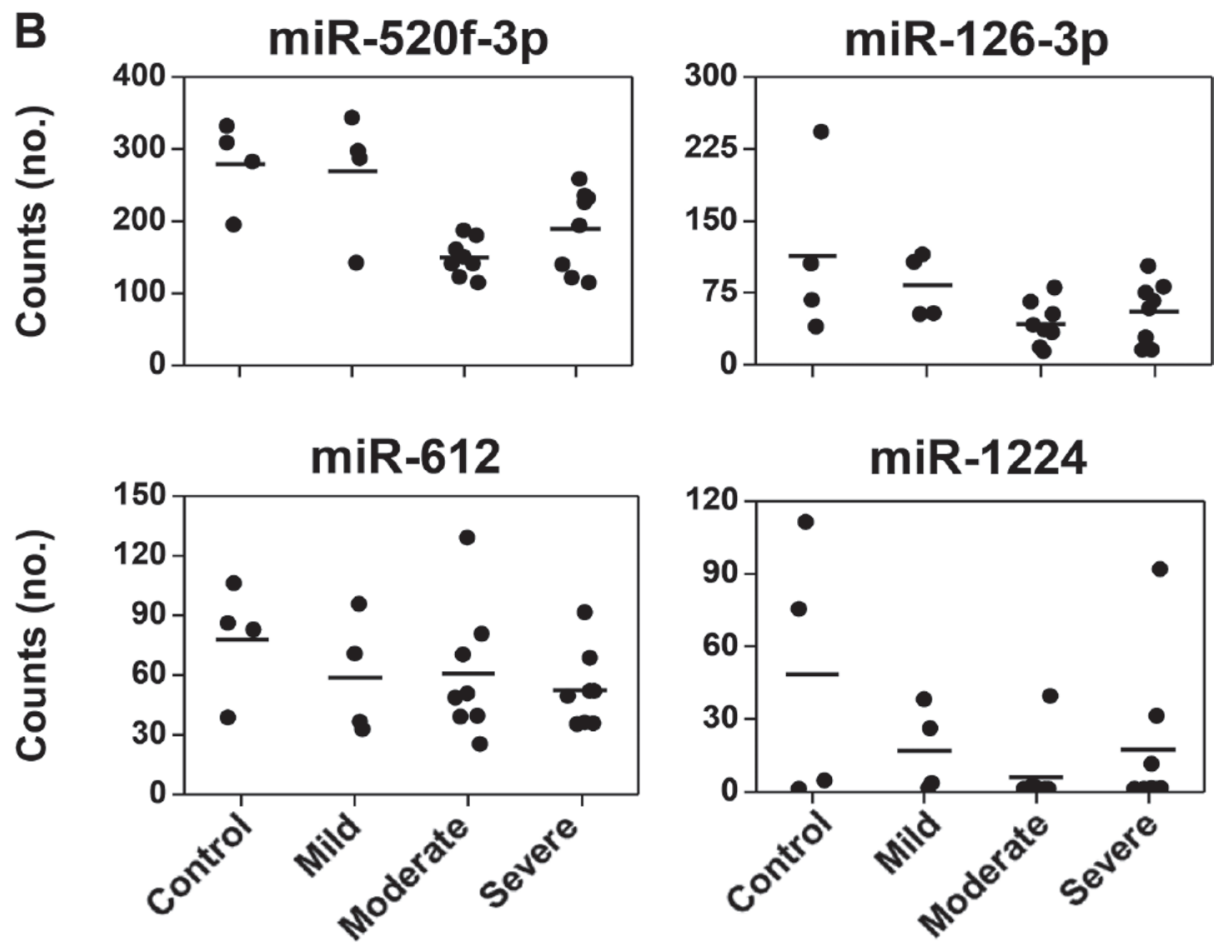

Downregulated miRNA in cattle serum

Figure 1. Identification of differentially expressed microRNA (miRNA) in the sera of cattle infected with Mycobacterium avium subspecies paratuberculosis (MAP) using nCounter (NanoString, Seattle, WA). Dot plot analysis showing miRNA counts as detected by the nCounter multiplex assay in the serum of cattle at different stages of Johne's disease. (A) Upregulated miRNA and (B) downregulated miRNA in animals with increased MAP infection compared with the control animals. The counts were obtained after normalizing against 3 spike-in controls. Mean counts of miRNA are denoted by horizontal lines, and each dot represents an individual animal. 
from the control and mild animals with a little overlap and accounted for $71.7 \%$ of the differences among the groups (Figure 4B).

The second model clearly separated the moderate from the control and mild infection groups $(P=0.009)$, whereas the severe infection group showed minimal overlap with the control and mild infection groups (Figure 4C). Overall, the data suggest that levels of expression of multiple miRNA in serum can help in differentiating MAP-infected animals from noninfected animals.

\section{DISCUSSION}

There is a large repertoire of miRNA in mammalian genomes. Some of these miRNA are highly conserved among different mammalian species and present in various body fluids (Siomi and Siomi, 2010). Circulating miRNA have been suggested to be upregulated in various infectious diseases and to have potential as novel biomarkers (Correia et al., 2017). Currently,

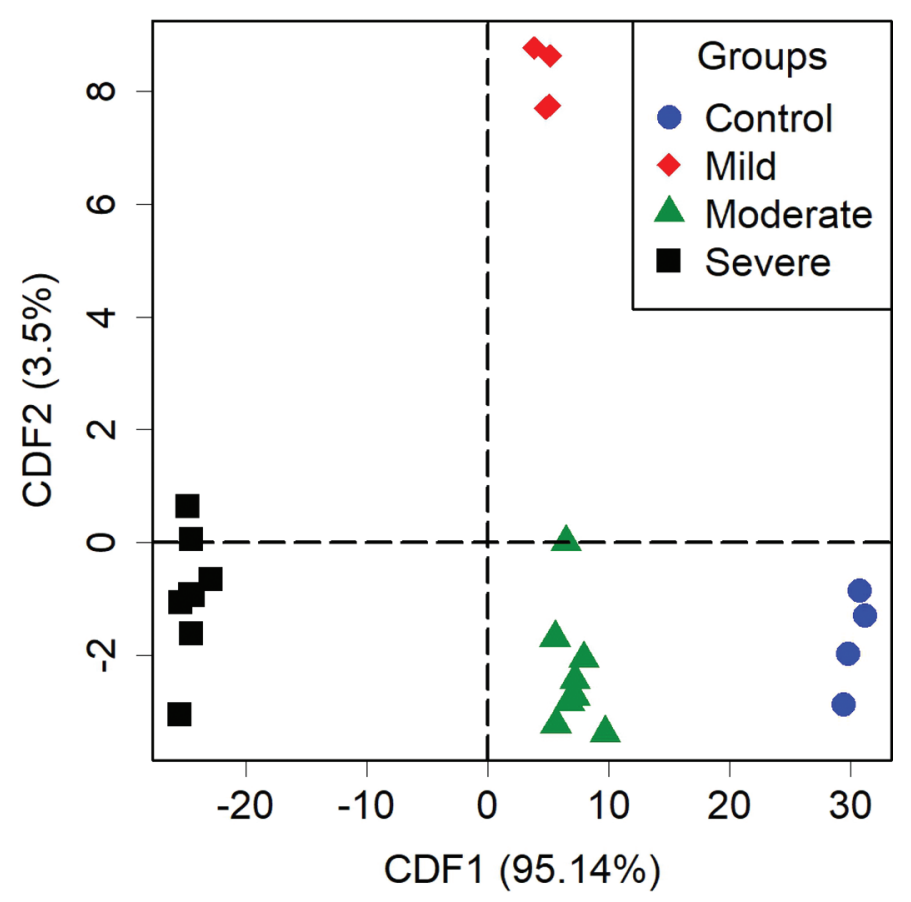

Figure 2. Canonical discrimination analysis of differentially expressed microRNA (miRNA) in the sera. Differential expressions of 20 miRNA were used for canonical discrimination analysis, which clearly separated the animals into 4 distinct clusters based on their disease severity: control, mild, moderate, and severe. The scores were calculated as canonical discriminant functions (CDF) and were represented in 2 dimensions: CDF1 and CDF2. Control and moderately and severely affected animals were clearly separated from each other in CDF1, whereas the mildly affected group was separated from the other groups in CDF2. Color version available online. quantitative PCR, microarray, and RNA sequencing are the methods of choice for studying the expression of miRNA (Pritchard et al., 2012; Tian et al., 2015). The NanoString nCounter technology applied here uses amplification-free sequence-specific probes-based hybridization to detect highly conserved miRNA. We used this technology to detect bovine miRNA using probe sets specific for human miRNA. In the current study, in silico analysis showed that around $21 \%$ of the human miRNA probes were of exact match to bovine miRNA. A previous study detected as low as $14 \%$ of miRNA in mouse serum with high confidence using 600 probe sets specific to mouse miRNA developed by the NanoString nCounter technology (Jacob et al., 2013). Hybridization-based detection of target miRNA is ensured by using large excess of sequence-specific probes, miRNA-specific tags, and bridging oligos with optimized melting temperature (Tm). The efficiency and specificity of the reaction are further monitored through each step by using control miRNA included in the sample preparation kit. Despite the technology's high specificity, 8 miRNA were detected with 1 - to 2 -nt difference compared with the human miRNA probes. It has been demonstrated that a mismatch of 1 to 2 nt can result in cross-hybridization of miRNA and probes (Mestdagh et al., 2014; Dennis et al., 2016). Thus, it is conceivable that the detection of 8 bovine miRNA with a 1- to 2-nt mismatch could be a result of cross-hybridization between highly homologous cattle miRNA and human miRNA probes. Nonetheless, these findings suggest that the NanoString nCounter technology is capable of detecting circulating miRNA in cattle and provide impetus to develop cattle-specific miRNA probes for bovine research.

The majority of miRNA detected have not been previously reported. Only 5 of the miRNA have been previously identified in cattle infected with MAP: miR-126, miR192, miR-223, miR-142, and miR-23a-3p (Farrell et al., 2015; Shaughnessy et al., 2015; Malvisi et al., 2016). A possible explanation is that previous studies used experimentally challenged cattle that might have a different miRNA profile compared with naturally infected animals, as were used here. This is supported by the differences in fecal shedding patterns between cattle naturally and experimentally infected with MAP, which suggests differences in their immune response to the infection (Mitchell et al., 2015). In addition to detecting known bovine miRNA in cattle serum, previously uncharacterized bovine miRNA were identified in the current study. Further research involving both naturally and experimentally infected animals will be required to confirm the miRNA profiles in these animals, including the role of the 13 previously uncharacterized miRNA described here. 


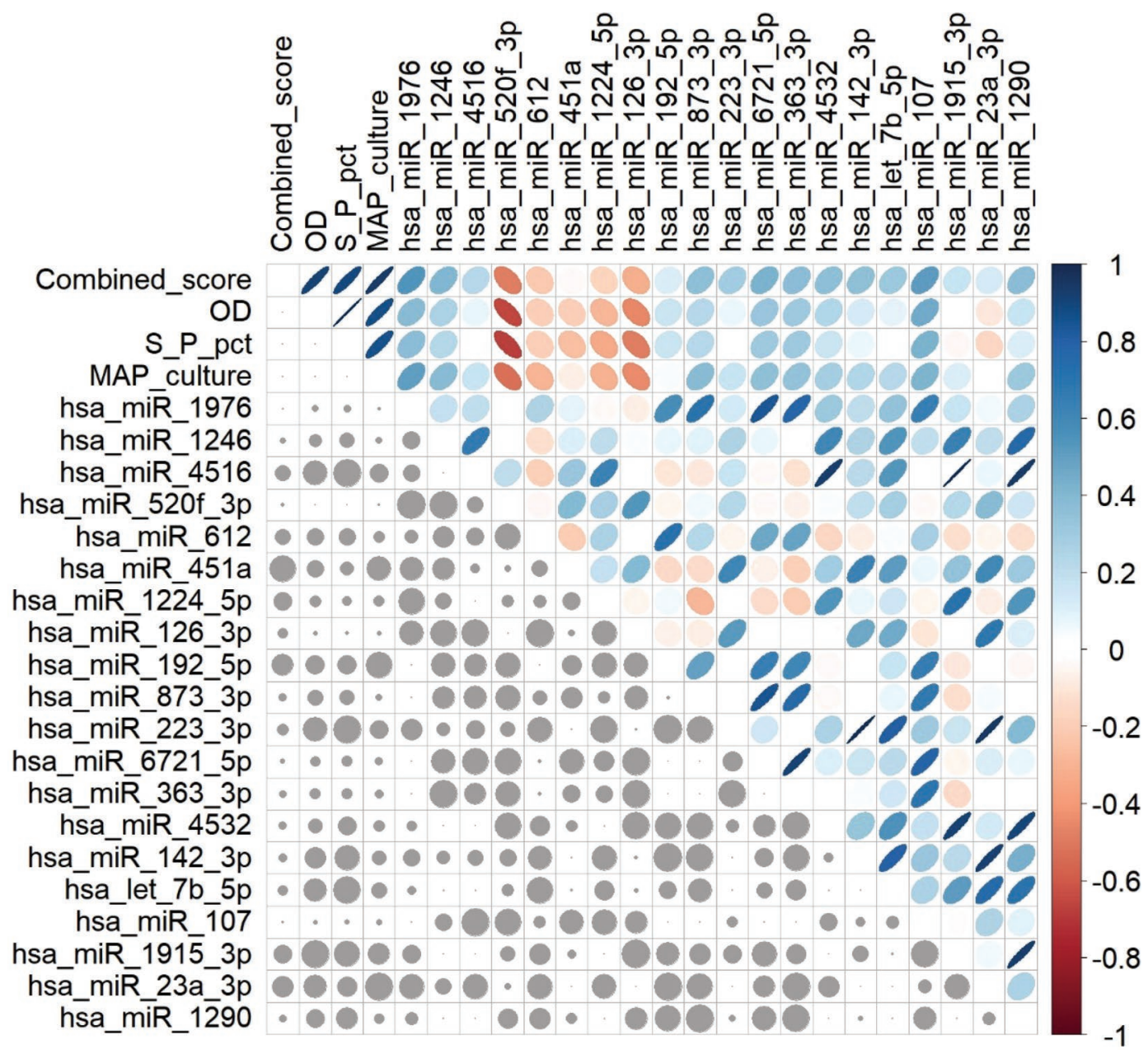

Figure 3. Pairwise correlations between differentially expressed microRNA (miRNA) and pathophysiological conditions. The expression levels of 20 miRNA were used to perform a correlation analysis with the pathophysiological conditions [combined histopathology score, optical density (OD), and S/P\% $100 \times$ (sample OD450 - negative control OD450)/(positive control OD450 - negative control OD450)] as well as Mycobacterium avium subspecies paratuberculosis (MAP) culture of the animals at various stages of the disease. The upper triangle shows the correlations, where blue ellipses indicate positive correlation and red ellipses indicate negative correlation. The lower triangle shows their significance; the smaller the circle, the higher the significance (i.e., the smaller the $P$-value). The range of pairwise correlations between histopathology scores and miRNA expressions is -0.6724 to 0.5577 , indicating some moderately large pairwise correlation between the 2 sets of variables.

Growing evidence suggests that miRNA are released from cells via exosomes as a result of infection to modulate gene expression in distant tissues or cells (Montecalvo et al., 2012; Turchinovich et al., 2013; Alexander et al., 2015). It is therefore possible that their level in body fluid could correlate with progression of disease. To date, no data are available on correlation of miRNA expression profile in MAP-infected tissues and circulating miRNA in cattle. A recent study demonstrated differential expression of 9 miRNA in the ileum tissue of calves experimentally infected with MAP in the intestine (Liang et al., 2016). But none of these miRNA were detected in the serum of MAP-infected cattle either in the current study or in previous studies (Farrell et al., 2015; Malvisi et al., 2016). One possible explanation for this difference in miRNA profile is that the animals in these studies were at different stages of infection and immune response. It is well known that MAP is a slow-growing bacterium and takes a long time to show signs in the infected animals (Koets et al., 2015; Fecteau, 2018). Further characterization of the miRNA profile of gut tissue and serum is needed to establish a link between tissue secreted and circulating miRNA in MAP-infected animals.

It has been suggested that miRNA participate in the regulation of immune responses during infection (Lee et al., 2014; Mehta and Baltimore, 2016). It has been demonstrated that expression of bta-miR-223 and btamiR-1246 is upregulated in the bovine mammary gland in response to Staphylococcus aureus and Streptococcus 


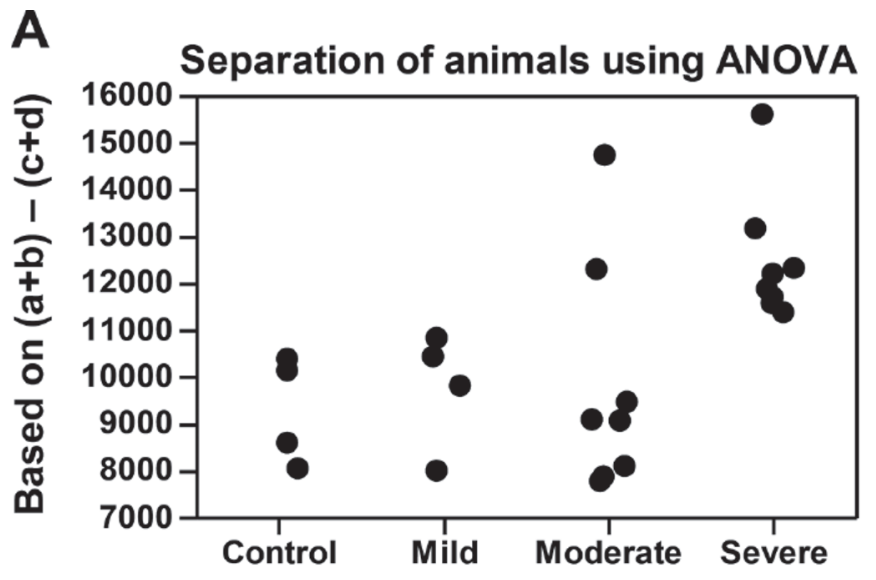

B

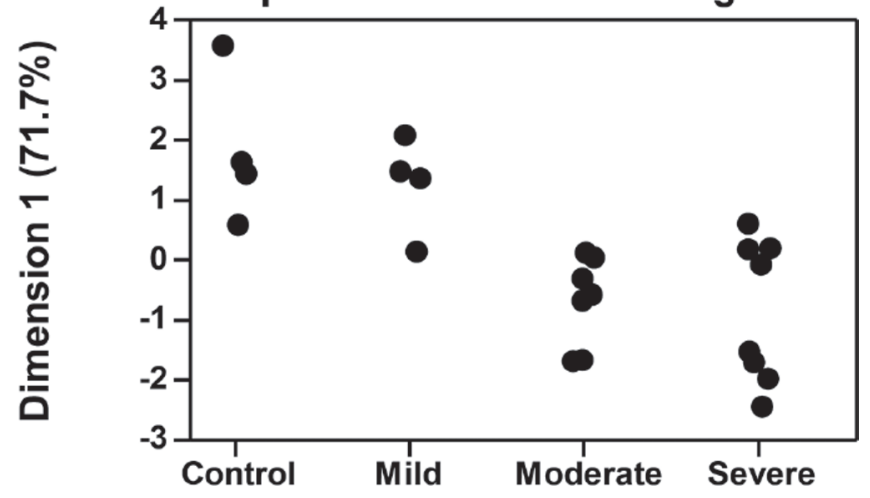

C

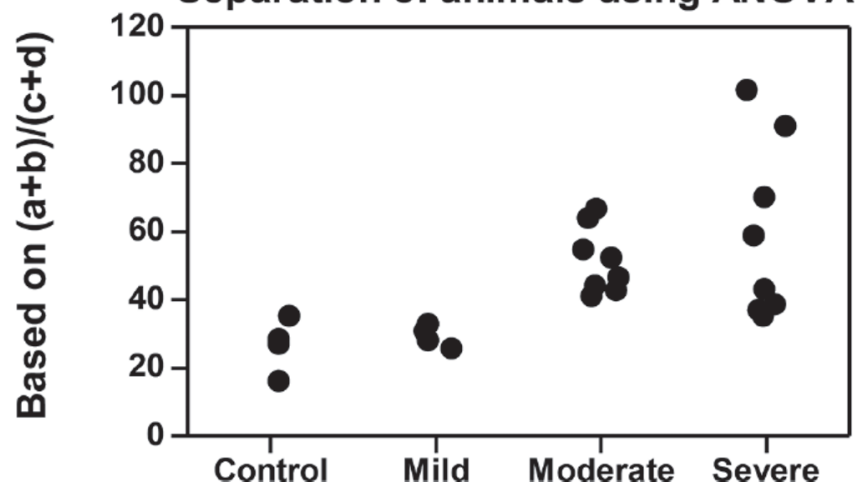

Figure 4. Development of diagnostic models based on expression of 4 microRNA (miRNA). The first model was generated using the difference in the expression of 4 miRNA (upregulated miR-1976 + miR-873-3p and downregulated miR-520f-3p + miR-126-3p) to examine the diagnostic potential of these 4 miRNA in a combination. The ability of this model to separate animals based on the status of disease severity was tested for significance using (A) ANOVA and (B) percentage separation statistics from canonical discrimination analysis (CDA) summaries. Differences among the groups were observed at $71.7 \%$ in CDF1. (C) The second based model was generated using the ratio of the 2 upregulated (miR-1976 + miR-873-3p) and 2 downregulated (miR-520f-3p + miR-126-3p) miRNA. Using ANOVA, this model significantly separated the moderate group from the mild and control groups, whereas little overlap of the latter 2 groups was observed with the severe group. uberis infection (Naeem et al., 2012; Li et al., 2015). A trend of increased expression for miR-223 (3.6-fold) was observed in the serum of severely infected animals compared with the controls. MicroRNA-223 has been reported to regulate differentiation and function of intestinal dendritic cells and macrophages (Zhou et al., 2015). On the other hand, increased levels of miR-1246 have been associated with inflammatory bowel disease and Crohn's disease in humans. Several studies have proposed a link between identification of MAP infection and Crohn's disease in humans (Liverani et al., 2014; Naser et al., 2014; McNees et al., 2015). Our results showed an increase in the levels of miR-1246 in the serum of severely infected animals. Given that the pathology of JD in cattle is similar to Crohn's disease in humans, it is conceivable that miR-1246 could be a potential marker for JD in cattle. Additionally, levels of miR-126-3p have been shown to increase in the serum of cattle infected with MAP (Farrell et al., 2015). This finding contradicts our observation in which a 2 -fold significant reduction was observed in the levels of miR126-3p in the animals severely infected with MAP compared with the controls. Further experiments will be required for deeper understating of the functionality of these differentially expressed miRNA in cattle infected with MAP.

Recent transcriptomics studies have revealed differential expression of a large number of mRNA and miRNA during infection and have proposed that their profile could be used as potential diagnostic biomarkers during infection (Hayes et al., 2014; Correia et al., 2017). The majority of these investigations have focused on human diseases, whereas relatively few investigations on infectious diseases in livestock have been reported. Maertzdorf et al. (2016) quantified expression of 281 genes and showed that a combination of only 4 of these genes can distinguish human tuberculosis patients from healthy individuals with $88 \%$ accuracy. Similarly, another study demonstrated that a combination of 15 differentially expressed miRNA in serum can diagnose active pulmonary TB in humans with $82 \%$ accuracy (Miotto et al., 2013). Although other studies reported differential expression of circulating miRNA in MAP-infected cattle (Farrell et al., 2015; Liang et al., 2016; Malvisi et al., 2016), to our knowledge no study has provided an approach to distinguish MAP-infected cattle from noninfected animals based on miRNA expression. Our model, using a combination of 4 differentially expressed miRNA (miR-1976, miR-873-3p, miR-520f-3p, and miR-126-3p), can distinguish moderate and severely naturally infected animals from healthy animals with $72 \%$ confidence. Given that this model is based on only 4 miRNA, a simple and cost-effective real-time PCRbased assay can be developed to measure expression 
of these 4 miRNA to diagnose MAP-infected animals. Further studies with a larger number of animals will be required to establish the suitability of these miRNA as diagnostic markers for JD.

\section{CONCLUSIONS}

This study reported detection of miRNA in cattle serum using sequence-specific probes for human miRNA in an amplification-free, hybridization-based direct digital counting platform. This study is the first to report differential expression of circulating miRNA in cattle using the NanoString nCounter technology. By comparing differential expression of miRNA we identified 4 miRNA that in combination have the potential to distinguish healthy animals from severely MAPinfected animals. This small set of 4 miRNA could offer an easy and cost-effective real-time PCR-based test for JD diagnosis. Overall, these findings indicate that probe-based detection of bovine miRNA is possible using NanoString nCounter technology and suggest that this approach can be applied to identify miRNA signatures to detect MAP infection at earlier stages but also as biomarkers for other infectious diseases in livestock.

\section{ACKNOWLEDGMENTS}

This work was supported by the AgResearch Curiosity Fund awarded to SKG, funded through the Strategic Science Investment Fund supported by the Ministry of Business, Innovation and Employment (Wellington, New Zealand).

\section{REFERENCES}

Alexander, M., R. Hu, M. C. Runtsch, D. A. Kagele, T. L. Mosbruger, T. Tolmachova, M. C. Seabra, J. L. Round, D. M. Ward, and R. M. O'Connell. 2015. Exosome-delivered microRNAs modulate the inflammatory response to endotoxin. Nat. Commun. 6:7321.

Camacho, C., G. Coulouris, V. Avagyan, N. Ma, J. Papadopoulos, K. Bealer, and T. L. Madden. 2009. BLAST+: Architecture and applications. BMC Bioinformatics 10:421.

Casas, E., G. Cai, L. A. Kuehn, K. B. Register, T. G. McDaneld, and J. D. Neill. 2016. Association of microRNAs with antibody response to Mycoplasma bovis in beef cattle. PLoS One 11:e0161651.

Clarke, C. J. 1997. The pathology and pathogenesis of paratuberculosis in ruminants and other species. J. Comp. Pathol. 116:217-261.

Conesa, A., P. Madrigal, S. Tarazona, D. Gomez-Cabrero, A. Cervera, A. McPherson, M. W. Szcześniak, D. J. Gaffney, L. L. Elo, X. Zhang, and A. Mortazavi. 2016. A survey of best practices for RNA-seq data analysis. Genome Biol. 17:13.

Correia, C. N., N. C. Nalpas, K. E. McLoughlin, J. A. Browne, S. V. Gordon, D. E. MacHugh, and R. G. Shaughnessy. 2017. Circulating microRNAs as potential biomarkers of infectious disease. Front. Immunol. 8:118.

Dennis, L., M. Rhodes, K. Maclean, and A. Kharkia. 2016. MicroRNA biomarker discovery and validation. Pages $1-7$ in White Paper. Vol. USPCP-PM0007. NanoString Technologies Inc., Seattle, WA.

Dong, H., Q. Gao, X. Peng, Y. Sun, T. Han, B. Zhao, Y. Liu, C. Wang, X. Song, J. Wu, and L. Yang. 2017. Circulating microRNAs as potential biomarkers for veterinary infectious diseases. Front. Vet. Sci. 4:186.

Farrell, D., R. G. Shaughnessy, L. Britton, D. E. MacHugh, B. Markey, and S. V. Gordon. 2015. The identification of circulating MiRNA in bovine serum and their potential as novel biomarkers of early Mycobacterium avium subsp. paratuberculosis infection. PLoS One 10:e0134310.

Fecteau, M.-E. 2018. Paratuberculosis in cattle. Vet. Clin. North Am. Food Anim. Pract. 34:209-222.

Friendly, M., and J. Fox. 2010. Candisc: R package for canonical discriminant analysis. Accessed Aug. 2, 2018. http://cran.r-project .org/web/packages/candisc.

Garcia, A. B., and L. Shalloo. 2015. Invited review: The economic impact and control of paratuberculosis in cattle. J. Dairy Sci. 98:5019-5039.

Geiss, G. K., R. E. Bumgarner, B. Birditt, T. Dahl, N. Dowidar, D. L. Dunaway, H. P. Fell, S. Ferree, R. D. George, T. Grogan, J. J. James, M. Maysuria, J. D. Mitton, P. Oliveri, J. L. Osborn, T. Peng, A. L. Ratcliffe, P. J. Webster, E. H. Davidson, L. Hood, and K. Dimitrov. 2008. Direct multiplexed measurement of gene expression with color-coded probe pairs. Nat. Biotechnol. 26:317325.

Griffiths-Jones, S. 2004. The microRNA registry. Nucleic Acids Res. 32(Suppl. 1):D109-D111.

Griffiths-Jones, S., H. K. Saini, S. van Dongen, and A. J. Enright. 2008. miRBase: Tools for microRNA genomics. Nucleic Acids Res. 36:D154-D158.

Gupta, S. K., B. J. Haigh, and T. T. Wheeler. 2016. Abundance of RNase4 and RNase5 mRNA and protein in host defence related tissues and secretions in cattle. Biochem. Biophys. Rep. 8:261-267.

Hayes, J., P. P. Peruzzi, and S. Lawler. 2014. MicroRNAs in cancer: Biomarkers, functions and therapy. Trends Mol. Med. 20:460-469.

He, L., and G. J. Hannon. 2004. MicroRNAs: Small RNAs with a big role in gene regulation. Nat. Rev. Genet. 5:522-531.

Jacob, N. K., J. V. Cooley, T. N. Yee, J. Jacob, H. Alder, P. Wickramasinghe, K. H. Maclean, and A. Chakravarti. 2013. Identification of sensitive serum microRNA biomarkers for radiation biodosimetry. PLoS One 8:e57603.

Jin, W., J. R. Grant, P. Stothard, S. S. Moore, and L. L. Guan. 2009. Characterization of bovine miRNAs by sequencing and bioinformatics analysis. BMC Mol. Biol. 10:90.

Koets, A. P., S. Eda, and S. Sreevatsan. 2015. The within host dynamics of Mycobacterium avium ssp. paratuberculosis infection in cattle: Where time and place matter. Vet. Res. 46:61.

Kozomara, A., and S. Griffiths-Jones. 2014. miRBase: Annotating high confidence microRNAs using deep sequencing data. Nucleic Acids Res. 42(D1):D68-D73.

Lawless, N., P. Vegh, C. O'Farrelly, and D. J. Lynn. 2014. The role of microRNAs in bovine infection and immunity. Front. Immunol. 5:611.

Lee, H.-M., D. T. Nguyen, and L.-F. Lu. 2014. Progress and challenge of microRNA research in immunity. Front. Genet. 5:178.

Li, R., C.-L. Zhang, X.-X. Liao, D. Chen, W.-Q. Wang, Y.-H. Zhu, X.H. Geng, D.-J. Ji, Y.-J. Mao, Y.-C. Gong, and Z.-P. Yang. 2015. Transcriptome microRNA profiling of bovine mammary glands infected with Staphylococcus aureus. Int. J. Mol. Sci. 16:4997-5013.

Li, S.-C., W.-C. Chan, L.-Y. Hu, C.-H. Lai, C.-N. Hsu, and W.-C. Lin. 2010. Identification of homologous microRNAs in 56 animal genomes. Genomics 96:1-9.

Liang, G., N. Malmuthuge, Y. Guan, Y. Ren, P. J. Griebel, and L. L. Guan. 2016. Altered microRNA expression and pre-mRNA splicing events reveal new mechanisms associated with early stage $M y-$ cobacterium avium subspecies paratuberculosis infection. Sci. Rep. 6:24964.

Liverani, E., E. Scaioli, C. Cardamone, P. Dal Monte, and A. Belluzzi. 2014. Mycobacterium avium subspecies paratuberculosis in the etiology of Crohn's disease, cause or epiphenomenon? World J. Gastroenterol. 20:13060-13070.

Maertzdorf, J., G. McEwen, J. Weiner, S. Tian, E. Lader, U. Schriek, H. Mayanja-Kizza, M. Ota, J. Kenneth, and S. H. E. Kaufmann. 
2016. Concise gene signature for point-of-care classification of tuberculosis. EMBO Mol. Med. 8:86-95.

Malvisi, M., F. Palazzo, N. Morandi, B. Lazzari, J. L. Williams, G. Pagnacco, and G. Minozzi. 2016. Responses of bovine innate immunity to Mycobacterium avium ssp. paratuberculosis infection revealed by changes in gene expression and levels of microRNA. PLoS One 11:e0164461.

McNees, A. L., D. Markesich, N. R. Zayyani, and D. Y. Graham. 2015. Mycobacterium paratuberculosis as a cause of Crohn's disease. Expert Rev. Gastroenterol. Hepatol. 9:1523-1534.

Mehta, A., and D. Baltimore. 2016. MicroRNAs as regulatory elements in immune system logic. Nat. Rev. Immunol. 16:279-294.

Mestdagh, P., N. Hartmann, L. Baeriswyl, D. Andreasen, N. Bernard, C. Chen, D. Cheo, P. D'Andrade, M. DeMayo, L. Dennis, S. Derveaux, Y. Feng, S. Fulmer-Smentek, B. Gerstmayer, J. Gouffon, C. Grimley, E. Lader, K. Y. Lee, S. Luo, P. Mouritzen, A. Narayanan, S. Patel, S. Peiffer, S. Rüberg, G. Schroth, D. Schuster, J. M. Shaffer, E. J. Shelton, S. Silveria, U. Ulmanella, V. Veeramachaneni, F. Staedtler, T. Peters, T. Guettouche, L. Wong, and J. Vandesompele. 2014. Evaluation of quantitative miRNA expression platforms in the microRNA quality control (miRQC) study. Nat. Methods 11:809-815.

Miotto, P., G. Mwangoka, I. C. Valente, L. Norbis, G. Sotgiu, R. Bosu, A. Ambrosi, L. R. Codecasa, D. Goletti, A. Matteelli, E. N. Ntinginya, F. Aloi, N. Heinrich, K. Reither, and D. M. Cirillo. 2013. miRNA signatures in sera of patients with active pulmonary tuberculosis. PLoS One 8:e80149.

Mitchell, R. M., Y. Schukken, A. Koets, M. Weber, D. Bakker, J. Stabel, R. H. Whitlock, and Y. Louzoun. 2015. Differences in intermittent and continuous fecal shedding patterns between natural and experimental Mycobacterium avium subspecies paratuberculosis infections in cattle. Vet. Res. 46:66.

Moldovan, L., K. E. Batte, J. Trgovcich, J. Wisler, C. B. Marsh, and M. Piper. 2014. Methodological challenges in utilizing miRNAs as circulating biomarkers. J. Cell. Mol. Med. 18:371-390.

Montecalvo, A., A. T. Larregina, W. J. Shufesky, D. Beer Stolz, M. L. G. Sullivan, J. M. Karlsson, C. J. Baty, G. A. Gibson, G. Erdos, Z. Wang, J. Milosevic, O. A. Tkacheva, S. J. Divito, R. Jordan, J. Lyons-Weiler, S. C. Watkins, and A. E. Morelli. 2012. Mechanism of transfer of functional microRNAs between mouse dendritic cells via exosomes. Blood 119:756-766.

Mortier, R. A. R., H. W. Barkema, and J. De Buck. 2015. Susceptibility to and diagnosis of Mycobacterium avium subspecies paratuberculosis infection in dairy calves: A review. Prev. Vet. Med. 121:189-198.

Naeem, A., K. Zhong, S. J. Moisá, J. K. Drackley, K. M. Moyes, and J. J. Loor. 2012. Bioinformatics analysis of microRNA and putative target genes in bovine mammary tissue infected with Streptococcus uberis. J. Dairy Sci. 95:6397-6408.

Naser, S. A., S. R. Sagramsingh, A. S. Naser, and S. Thanigachalam. 2014. Mycobacterium avium subspecies paratuberculosis causes Crohn's disease in some inflammatory bowel disease patients. World J. Gastroenterol. 20:7403-7415.

Nugent, G., E. J. Whitford, J. C. Hunnam, P. R. Wilson, M. I. Cross, and G. W. de Lisle. 2011. Mycobacterium avium ssp. paratuberculosis infection in wildlife on three deer farms with a history of Johne's disease. N. Z. Vet. J. 59:293-298.
Pritchard, C. C., H. H. Cheng, and M. Tewari. 2012. MicroRNA profiling: Approaches and considerations. Nat. Rev. Genet. 13:358-369.

Rohart, F., B. Gautier, A. Singh, and K.-A. Lê Cao. 2017. mixOmics: An $\mathrm{R}$ package for 'omics feature selection and multiple data integration. PLOS Comput. Biol. 13:e1005752.

Shaughnessy, R. G., D. Farrell, K. Riepema, D. Bakker, and S. V. Gordon. 2015. Analysis of biobanked serum from a Mycobacterium avium subsp paratuberculosis bovine infection model confirms the remarkable stability of circulating miRNA profiles and defines a bovine serum miRNA repertoire. PLoS One 10:e0145089.

Shu, D., S. Subharat, D. N. Wedlock, D. Luo, G. W. de Lisle, and B. M. Buddle. 2011. Diverse cytokine profile from mesenteric lymph node cells of cull cows severely affected with Johne's disease. Clin. Vaccine Immunol. 18:1467-1476.

Siomi, H., and M. C. Siomi. 2010. Posttranscriptional regulation of microRNA biogenesis in animals. Mol. Cell 38:323-332.

Smith, R. L., M. A. Al-Mamun, and Y. T. Gröhn. 2017. Economic consequences of paratuberculosis control in dairy cattle: A stochastic modeling study. Prev. Vet. Med. 138:17-27.

Sun, J., K. Aswath, S. G. Schroeder, J. D. Lippolis, T. A. Reinhardt, and T. S. Sonstegard. 2015. MicroRNA expression profiles of bovine milk exosomes in response to Staphylococcus aureus infection. BMC Genomics 16:806.

Taxis, T. M., F. V. Bauermann, J. F. Ridpath, and E. Casas. 2017 Circulating microRNAs in serum from cattle challenged with bovine viral diarrhea virus. Front. Genet. 8:91.

Tian, T., J. Wang, and X. Zhou. 2015. A review: microRNA detection methods. Org. Biomol. Chem. 13:2226-2238.

Tsang, H.-F., V. W. Xue, S.-P. Koh, Y.-M. Chiu, L. P.-W. Ng, and S.C. C. Wong. 2017. NanoString, a novel digital color-coded barcode technology: Current and future applications in molecular diagnostics. Expert Rev. Mol. Diagn. 17:95-103.

Turchinovich, A., T. Samatov, A. Tonevitsky, and B. Burwinkel. 2013. Circulating miRNAs: Cell-cell communication function? Front. Genet. 4:119.

Wang, H., C. Horbinski, H. Wu, Y. Liu, S. Sheng, J. Liu, H. Weiss, A. J. Stromberg, and C. Wang. 2016. NanoStringDiff: A novel statistical method for differential expression analysis based on NanoString nCounter data. Nucleic Acids Res. 44:e151.

Wang, K., Y. Yuan, J.-H. Cho, S. McClarty, D. Baxter, and D. J. Galas. 2012. Comparing the microRNA spectrum between serum and plasma. PLoS One 7:e41561.

Whitlock, R. H., and C. Buergelt. 1996. Preclinical and clinical manifestations of paratuberculosis (including pathology). Vet. Clin. North Am. Food Anim. Pract. 12:345-356.

Whittington, R. J., I. Marsh, S. McAllister, M. J. Turner, D. J. Marshall, and C. A. Fraser. 1999. Evaluation of modified BACTEC 12B radiometric medium and solid media for culture of Mycobacterium avium ssp. paratuberculosis from sheep. J. Clin. Microbiol. 37:1077-1083.

Zhang, J., S. Li, L. Li, M. Li, C. Guo, J. Yao, and S. Mi. 2015. Exosome and exosomal microRNA: trafficking, sorting, and function. Genomics Proteomics Bioinformatics 13:17-24.

Zhou, H., J. Xiao, N. Wu, C. Liu, J. Xu, F. Liu, and L. Wu. 2015. MicroRNA-223 regulates the differentiation and function of intestinal dendritic cells and macrophages by targeting $\mathrm{C} / \mathrm{EBP} \beta$. Cell Reports 13:1149-1160. 